\title{
Pharmacological management of psychiatric disorders in pregnancy complicated by diabetics
}

\author{
Yatan Pal Singh Balhara, Rohit Verma ${ }^{1}$, Bharti Kalra ${ }^{2}$ \\ Department of Psychiatry, National Drug Dependence Treatment Centre, All India Institute of Medical Sciences, New Delhi, ${ }^{1}$ Psychiatry, \\ Lady Hardinge Medical College and SSK Hospital, New Delhi, ${ }^{2}$ Gynaecology, Bharti Hospital and B.R.I.D.E., Karnal, India
}

\section{A B S T R A C T}

Pregnancy has been identified as a biological, psychological and social stressor that predisposes women to emergence/re-emergence of psychiatric disorders. Effective pharmacological treatment is available for various psychiatric disorders. However, management of psychiatric disorders during pregnancy requires careful selection of the pharmacotherapeutic agent. Management of psychiatric disorders in pregnancy among diabetics brings in an additional dimension. The psychotropic medicines have been found to impact blood glucose levels and can interfere with diabetes management. This article reviews the available evidence on the use of psychotropic medicines in antenatal women with diabetes.

Key words: Diabetes mellitus, management, psychiatric disorders, depression, anxiety, psychosocial management

\section{INTRODUCTION}

Pregnancy has been identified as a biological, psychological and social stressor that predisposes women to emergence/ re-emergence of psychiatric disorders. Altered hormonal balance, stress of childbirth and awareness of the increased responsibility of motherhood coupled with the expectations of the family members put women at an increased risk of emergence of psychiatric disorders. Also, there is an increased risk of relapse of the preexisting psychiatric disorders.

Effective pharmacological treatment is available for various psychiatric disorders. However, management of psychiatric disorders during pregnancy requires careful selection of the pharmacotherapeutic agent. The same caution needs to be exercised during breast feeding as well as almost

\begin{tabular}{|l|l|}
\hline \multicolumn{2}{|c|}{ Access this article online } \\
\hline Quick Response Code: & Website: \\
\hline & www.joshd.net \\
\hline & \\
\hline
\end{tabular}

all psychotropic medications are excreted in breast milk, although to a variable extent.

Management of psychiatric disorders in pregnancy among diabetics brings in an additional dimension. The psychotropic medicines have been found to impact blood glucose levels and can interfere with diabetes management.

Finally, interactions between oral hypoglycemic agents, insulin and psychotherapeutic agents need to be kept in mind while deciding on the appropriate therapy for the management of psychiatric disorders in pregnancy among diabetics.

Medications used for the management of psychiatric disorders can be broadly classified as anti-depressants, antipsychotics and mood stabilizers. Although this classification is not all inclusive and is considered over simplistic, it continues to be used widely. Modern psychiatric practice involves use of many more medications, including benzodiazepines and centrally acting anti-cholinergics, among others.

\section{Impact of psychotropic medications on glycemia control Anti-depressants \\ The impact of anti-depressant medications (used for management of depressive disorders; anxiety disorders}

Corresponding Author: Dr. Yatan Pal Singh Balhara, Department of Psychiatry, National Drug Dependence Treatment Centre (NDDTC), All India Institute of Medical Sciences (AlIMS), New Delhi - 110 029, India. E-mail: ypsbalhara@gmail.com 
including Obsessive Compulsive Disorder, phobias, panic disorder, stress-related disorders; somatoform disorders) on glucose levels and outcome of diabetes is rather complex. It has been suggested that tricyclic antidepressants (TCAs) may increase the serum concentrations of glucose, resulting in an increased craving for sweets. ${ }^{[1]}$ The studies involving unselective monoaminergic inhibitors (TCAs such as amitriptiline, imipramine and clomipramine) have come with mixed results for impact on glucose metabolism. While some studies have failed to find any significant impact of these agents on glucose metabolism, ${ }^{[2-4]}$ others have documented altered glucose metabolism following use of these medicines. ${ }^{[5,6]} \mathrm{A}$ large body of literature recommends against the use of TCAs as a first-line treatment for this population based on the findings from observational studies and associated cardiovascular and anti-cholinergic side-effects. Nortriptyline has been found to have either no effect or an increase in the blood glucose levels. ${ }^{[1,7]}$ Mianserine has also been associated with an increase in the blood glucose (postprandial) levels. ${ }^{[8]}$ Among the selective serotonin reuptake inhibitors (SSRIs), fluoxetine, citalopram, escitalopram, paroxetine and sertraline have been found to be associated with either no change or a reduction in the levels of blood glucose..$^{[9-17]}$ Fluoxetine remains the most widely studied and is consistently associated with reduced blood glucose levels among the SSRIs. ${ }^{[17]}$ Venlafaxine and duloxetine, serotonine and norepinephrine reuptake inhibitors have been found to have either no effect or an increase in the glucose levels. ${ }^{[9,18,19]}$ Duloxetine has been approved for diabetic neuropathy. Monoamine oxidase inhibitors are associated with lowering of blood glucose levels, especially among those who respond to these medications. ${ }^{[20]}$ Mirtazapine has not been associated with an increase in blood glucose levels. Its use has been found to have either no change or an improvement in glycemia control. ${ }^{[21,22]}$

However, it must be mentioned that these observations are based on short to medium duration studies. Also, the studies included were heterogeneous with regard to the methodology. There is some evidence that suggests that recent use of amitriptyline, fluvoxamine, paroxetine and venlafaxine among long-term users (>24 months) is associated with an increased risk of emergence of diabetes. Moreover, the research examining the use of SSRIs in the treatment of diabetic neuropathy is limited.

Finally, only a handful of randomized controlled trials have assessed the impact of anti-depressants on diabetes control among individuals with co-morbid depression. While trials involving nortriptyline and fluoxetine (one each) have found an improvement in the level of depression, the beneficial effects on glucose control were not observed. In fact, use of nortriptyline was associated with worsening of glycemia control..$^{[1,23]}$ Use of bupropion has been reported to have a beneficial effect on both depression and glycemia control. ${ }^{[24]}$ One trial with sertraline found a significantly lower glycemia control when it was used as prophylaxis against emergence of depression. ${ }^{[14]}$ The Pathways study (Pathways Collaborative Care Intervention, using a stepped-care approach including use of anti-depressants) showed a reduced severity of depression but no change in glycemia control. ${ }^{[25]}$

\section{Anti-psychotics}

Among the anti-psychotics, the newer ones (also called as atypical anti-psychotics or serotonine dopamine antagonists), as a group, are more likely to lead to impaired glycemia control as compared with the typical antipsychotics (dopamine antagonists). Within the atypical anti-psychotic group, clozapine and olanzapine have a high propensity for this effect while ziprasidone and aripiprazole are associated with a lower risk. The risk is intermediate for risperisdone and quetiapine. The risk is low with atypical anti-psychotics, especially the highpotency ones (e.g., haloperidol).

\section{Mood stabilizers}

Mood stabilizers are another class of drugs commonly prescribed for the management of psychiatric disorders such as bipolar disorders. Among the various mood stabilizers, two of the most commonly used agents - lithium and sodium valproate- are associated with a high risk of impaired glycemia control. ${ }^{[26,27]}$ The risk is low with topiramate, which is actually associated with a lowering of the blood glucose levels. ${ }^{[28]}$ The literature is limited to draw meaningful conclusions on the impact of other mood stabilizers on glycemia control.

\section{Psychotropic medications and fetal development}

Another important consideration to be made while managing psychiatric disorders using psychotropics among pregnant women with diabetes is the impact of these medications on the developing fetus. Other issues that should be taken into consideration include the risk of behavioral teratogenesis and perinatal syndrome. Also, the clinicians need to be aware of the secretion of these medications in breast milk that can reach the breast-fed infant.

\section{Anti-depressants}

Among the anti-depressants, neither the TCAs nor fluoxetine (an SSRI) has been associated with major teratogenic effects. ${ }^{[29-32]}$ There are fewer data on other SSRIs such as paroxetine, sertraline, citalopram and escitalopram. There are reports associating the use of paroxetine during 
pregnancy and cardiac malformations in the newborn, ${ }^{[33]}$ although these findings have been disputed and there are reports that have failed to find such associations. ${ }^{[34,35]}$ The data is even sparser for newer anti-depressants such as venlafaxine, nefazodone, mirtazapine, duloxetine and bupropion. However, a statistically significant increase in congenital anomalies has not been observed in studies using these medications. ${ }^{[36]}$

Also, there is no evidence of behavioral teratogenicity with these agents in studies up to 7 years of follow-up. ${ }^{[37]}$ While most of these agents have been reported to be associated with perinatal syndromes, the effects appear to be mild and transient. Also, a causal association remains to be proven as most of the literature is from case reports. Some of the clinical features of the neonatal syndrome observed among neonates exposed to TCAs during the intrauterine period include jitteriness, irritability and, rarely, convulsions. Poor neonatal adaptation characterized by tachypnea, hypoglycemia, temperature instability, irritability, a weak or absent cry and seizures has been reported during the immediate neonatal days among infants exposed to SSRIs. ${ }^{[38]}$

Limited information is available about the possible longterm effects of anti-depressant exposure in utero, although few studies including SSRISs (at 6-9 months) and TCAs (in childhood) have failed to find adverse neuro-cognitive effects. ${ }^{[37]}$ Limited data on the use of monoamine oxidase inhibitors are not reassuring, and use of these agents is not recommended during pregnancy. ${ }^{[36]}$

\section{Anti-psychotics}

Among the anti-psychotics, there is a stronger body of evidence on typical anti-psychotics as they are in use for a longer duration. ${ }^{[39]}$ Although there are case reports linking haloperidol use during pregnancy with birth defects, larger cases series have failed to find such an association. ${ }^{[39]}$ It has been shown to be free of congenital malformations with first-trimester exposure and is a preferred agent during pregnancy. Studies on phenothiazines (e.g., chlorpromazine) have concluded that there is no increase in morphological or developmental abnormalities associated with them. ${ }^{[40]}$ However, transient perinatal syndrome, characterized by floppy infants, withdrawal symptoms such as increased irritability, hypotonicity and hypertonicity, and underdeveloped reflexes, have been reported in infants who have been exposed to different low-potency anti-psychotics in utero. Additionally, it is important to keep in mind the common co-prescription of centrally acting anti-cholinergic medication with high-potency typical antipsychotics to manage extrapyramidal side-effects. There are reports of possible teratogenicity associated with the use of anticholinergic drugs in combination with antipsychotics. The consensus is that they should be avoided when possible and should be used in the lowest possible dosage when prescribed.

In spite of a growing number of reported cases of women completing their pregnancy while taking clozapine, olanzapine, risperidone or quetiapine without any detrimental effects to the newborn, there is a lack of systematic studies exploring such an association. ${ }^{[41]}$ All second-generation anti-psychotics (with the exception of clozapine that comes in category B) come under the FDA category $\mathrm{C}$ of drug safety during pregnancy.

\section{Mood stabilizers}

Two of the most commonly used mood stabilizers, lithium ${ }^{[42]}$ and valproate, ${ }^{[43]}$ have been found to be associated with teratogenesis and organ dysgenesis following in utero exposure. Lithium use is associated with an increased incidence of Ebstein's anomaly as compared with the general population, although the absolute risk is small. Also, lithium has not been found to be associated with an increased risk of neurobehavioral teratogenicity. The most common toxicity effect in an offspring exposed to lithium during labor is the "floppy baby" syndrome, characterized by cyanosis and hypotonicity.

Use of valproate and carbamazepine during the first trimester is associated with increased rates of neural tube defect. ${ }^{[39]}$ Neonatal complications associated with valproate use include heart rate decelerations and withdrawal symptoms of irritability, jitteriness, feeding difficulties and abnormal tone.

The teratogenic potential of other mood stabilizers such as lamotrigine, gabapentin and topiramate is not well documented and remains unclear. ${ }^{[39]}$ However, there is some consensus emerging that lamotrigine is not associated with an increased risk of teratogenicity, including behavioral teratogenicity, when used during pregnancy.

\section{Other medicines}

Benzodiazepines are also commonly prescribed for the management of psychiatric disorders. These are sued as either adjunct (e.g., for insomnia in depressive disorders) or even main line of management for certain disorders (life alcohol withdrawal, anxiety disorders such as panic attacks). Benzodiazepines used in the first trimester have been linked to an increased risk of oral cleft and congenital malformations of the central nervous system and the urinary tract. ${ }^{[39]}$ These can also lead to neonatal withdrawal symptoms, respiratory depression, hypothermia, feeding 
difficulties and muscular hypotonia in the newborn. Use of high-potency compounds is expected to be preferable with regard to these effects as these have shorter half-lives and are less likely to cause sedation.

Use of beta-blockers (such as propranolol to manage anxiety symptoms and akathesia associated with antipsychotic medications) during pregnancy is not associated with an increase in congenital malformations.

Table 1 has grouped various anti-depressants, antipsychotics and mood stabilizers for fetal safety (based on US FDA category) and impact on glycaemia control. It must be noted that these groupings are based on the accessible evidence. The evidence continues to be limited for many medications and at times there are contradictory reports as well. It is strongly recommended to update oneself with the latest literature before finalizing the management plan. Also one needs to take into consideration other side effects associated with use of these medications while making the management plan.

\section{Psychotropic medications and breast feeding}

The postpartum period is associated with a change in the body dynamics. Issues such as change in body fluid distribution and gradual return to the prepregnancy state alter the pharmacokinetics of some of the psychotropic medications and need to be looked into carefully. Also, most of the psychotropic medications are excreted in breast milk as well. However, it is not possible to advise on the acceptable milk/plasma ratios or safe doses because of the lack of such data. It is advisable to monitor the breast-fed infant for any possible adverse effects such as drowsiness, hypotonia, rigidity, tremor and withdrawal symptoms if the mother is on psychotropics.

\section{Anti-depressants}

Studies of tricyclic anti-depressants in breast feeding have shown that drug concentration in breast milk is approximately the same as that in maternal plasma. Amitriptyline, imipramine, clomipramine and nortryptiline were found to not have significant serum levels in infants, and their use was not associated with any adverse events in breast-fed infants of mothers using these medicines across different studies. ${ }^{[44]}$ However, some TCAs such as doxepin have a long-acting metabolite and can get accumulated in infants. ${ }^{[45]}$

Among the SSRIs, sertraline has been recommended in the USA as the first-line treatment for breast-feeding mothers. This is because of the undetectable serum levels of sertraline and cumulative research evidence indicating

\begin{tabular}{|c|c|c|c|}
\hline \multirow{2}{*}{$\begin{array}{l}\text { Adverse effect on developing } \\
\text { fetus/new born based on } \\
\text { FDA category }\end{array}$} & \multicolumn{3}{|c|}{ Impact on glycemia control } \\
\hline & Little impact & Intermediate impact & High impact \\
\hline \multicolumn{4}{|l|}{ Anti-depressants } \\
\hline \multirow{5}{*}{ FDA category C } & Fluoxetine $^{\wedge}$ & & Venlafaxine $^{*}$ \\
\hline & Sertraline $^{\wedge}$ & & Fluvoxamine $^{*}$ \\
\hline & Citalopram^ & & \\
\hline & Escitalopram^ & & \\
\hline & Bupropion^@ & & \\
\hline \multirow[t]{3}{*}{ FDA category D } & & & TCAs (Amitriptyline, Nortriptyline) \\
\hline & & & MAOI \\
\hline & & & Paroxetine ${ }^{\#}$ \\
\hline \multicolumn{4}{|l|}{ Anti-psychotics } \\
\hline FDA category $B$ & & & Clozapine \\
\hline \multirow[t]{3}{*}{ FDA category C } & $\begin{array}{l}\text { High-potency typical anti-psychotics } \\
\text { (e.g., Haloperidol) }\end{array}$ & Risperidone $^{@}$ & Olanzapine ${ }^{@}$ \\
\hline & Aripiprazole ${ }^{@}$ & Quetiapine@ & \\
\hline & Ziprasidone ${ }^{@}$ & & \\
\hline Undetermined category & $\begin{array}{l}\text { Low-potency typical anti-psychotics } \\
\text { (e.g., Chlorpromazine) }\end{array}$ & & \\
\hline \multicolumn{4}{|l|}{ Mood stabilizers } \\
\hline FDA category $C$ & Lamotrigine@ & & Lithium \\
\hline FDA category D & Topiramate $^{\wedge}$ & & Valproate \\
\hline
\end{tabular}

Based on the available literature (literature inadequate for many medicines; lack of controlled trials)

^Favorable impact on blood glucose levels (reduction); associated with self-limiting neonatal syndrome @Limited literature on fetal safety

*Associated with emergence of diabetes on long-term use ( $>24$ months)

\$Associated with neonatal syndrome; additional cardiovascular and anti-cholinergic side-effects; additional cardiovascualr mia control in spite of improvement in depression.

\#Some reports of cardiac malformations; not observed across all studies.

FDA - The U.S. Food and Drug Administration 
no significant adverse effects on the infant. ${ }^{[46]}$ Similarly, most of the studies on fluoxetine have also reported the levels in infant serum to be very low or undetectable. ${ }^{[4]}$ However, long half-life and presence of an active metabolite make it less favorable for use. Similarly, paroxetine and escitalopram (limited evidence) use has not been found to be associated with adverse effects in infants. Citalopram also has not been associated with adverse effects in infants. However, its use is not recommended as its concentration in milk has been found to higher than that in plasma. ${ }^{[47]}$ There is limited literature on venlafaxine and mirtazapine, but no adverse effects have been associated with their use. The information on bupropion, trazodone and duloxetine is limited. ${ }^{[44]}$ However, one case report on bupropion reported the emergence of seizures in an infant..$^{[48]}$

\section{Anti-psychotics}

Typical anti-psychotics have been reported to be safe during breast feeding. ${ }^{[49]}$ Their levels in maternal milk and infant serum have been found to be low. Also, these have not been associated with any adverse effects in the infants. However, there have been reports of drowsiness, lethargy and possible gastrointestinal complaints.

Although there are no reports on adverse effects due to the use of olanzapine and risperidone by breast-feeding mothers, it has been recommended to consider the riskbenefit analysis before their prescription. ${ }^{[49]}$ Reports have suggested that clozapine accumulates in breast milk and its use is contraindicated during breast feeding. ${ }^{[50]}$ There is limited information on amisulpride and quetiapine to make any favorable recommendations for their use.

\section{Mood stabilizers}

The levels of lithium in breast milk levels have been found to be high. Adverse effects reported in infants include cyanosis, hypotonia, heart murmur, electrocardiogram changes, lethargy and hypothermia. ${ }^{[51]}$ Also, infants may be more susceptible to both dehydration and lithium toxicity due to their immature kidney function and the potential for rapid dehydration. Lithium is contraindicated during breast feeding. Use of valproate and carbamazepine has been reported to be compatible with breast feeding. ${ }^{[49]}$ However, transient hepatic toxicity such as hyperbilirubinemia and high concentrations of gamma glutamyl transferase has been reported in neonates exposed to carbamazepine during breast-feeding.

\section{Other medicines}

Benzodiazepines with shorter half-lives (i.e., lorazepam, alprazolam and oxazepam) have been found to be very low in breast milk. Repeated doses of long-acting benzodiazepines can produce lethargy, poor suckling and weight loss, and infants need to be monitored. Benzodiazepines taken by the mother during pregnancy should be withdrawn with caution to avoid withdrawals in the infants who might have developed physical dependence to these medicines during in utero exposure. ${ }^{[49]}$

Zaleplon and zopiclone should be avoided because they are excreted in breast milk. However, zolpidem is accepted as compatible with breast feeding because of its low lipophilic properties and rapid excretion.

\section{Drug interactions}

Many SSRIs and other anti-depressants impact the cytochrome enzyme systems. Because of the inhibition of the cytochrome P450 (CYP) 3A4 isoenzyme, some of the SSRIs may alter the metabolism of certain oral hypoglycemics (i.e., thiazolidinedione pioglitazone, meglitinides, repaglinide and nateglinide). Therefore, coprescription of nefazodone, fluoxetine and fluvoxamine carry a risk of problems with hypoglycemia. Also, inhibition of the CYP 2C9 isoenzyme by fluoxetine, fluvoxamine or sertraline would also potentially interfere with the CYP 2C9 metabolism of the sulfonylureas tolbutamide and glimepiride.

\section{Modified electroconvulsive therapy (MECT)}

MECT is also used as a treatment modality in the treatment of psychiatric disorders. Reported complications of MECT during pregnancy are uncommon and transient. ${ }^{[52]}$ Even the cases reported to have uterine contractions during MECT did not progress to premature labor. However, one needs to be cautious about the use of premedication and the anesthetic agent used during the procedure. Atropine can reduce beat-to-beat variability in the fetal heart rate and fetal tachycardia. Use of anticholinergic agents can decrease the esophageal sphincter tone, increasing the risk of gastric reflux. The patient can be intubated to prevent these complications.

Combination of pharmacological interventions with nonpharmacological interventions

Nonpharmacological interventions offer an excellent alternative/add-on intervention in the management of psychiatric disorders among pregnant women having diabetes. Not only are these interventions free from any teratogenic or behavioral adverse effects on the new born but they are also unlikely to impact the glycemia control. Many patients with a mild-to-moderate intensity of symptoms can be treated by psychosocial approaches. Even for those with more a severe form of disorders, use of psychological therapies as adjunct intervention can help reduce the dose of medications and improve the compliance and adherence to medications. Various 
psychotherapeutic interventions available for this purpose include cognitive behavioral therapy, interpersonal psychotherapy, supportive psychotherapy, exposure and response prevention and community-based behavioral intervention, among others. ${ }^{[38]}$ These can be administered in individual or group sessions.

While deciding the management of psychiatric disorders in a pregnant women having diabetes, it is important to take into consideration different factors. This includes assessment of the risk of relapse based on a past history of illness (in terms of duration, severity, treatment response, etc.), availability of interventions, availability and ease of follow-up with mental health professionals, individual and family concerns and choices, stage of pregnancy and quality of diabetes control. It is advisable to weigh the risks and benefits of withholding/delaying the treatment vis-à-vis the risks and benefits on introduction of the treatment for psychiatric disorders while finalizing the management plan.

\section{REFERENCES}

1. Lustman PJ, Griffith LS, Clouse RE, Freedland KE, Eisen SA, Rubin $\mathrm{EH}$, et al. Effects of nortriptyline on depression and glycemic control in diabetes: Results of a double-blind, placebocontrolled trial. Psychosom Med 1997;59:241-50.

2. Moosa MY, Panz VR, Jeenah FY, Joffe BI. African women with depression: The effect of imipramine and fluoxetine on body mass index and leptin secretion. J Clin Psychopharmacol 2003;23:549-52.

3. Ghaeli P, Shahsavand E, Mesbahi M, Kamkar MZ, Sadeghi M, Dashti-Khavidaki S. Comparing the effects of 8-week treatment with fluoxetine and imipramine on fasting blood glucose of patients with major depressive disorder. J Clin Psychopharmacol 2004;24:386-8.

4. Langer G, Karobath M, Sieghart W, Aschauer H, Placheta P, Spona J. Effects of antidepressant treatment with clomipramine on hormonal responses to thyrotropin-releasing hormone and insulin-induced hypoglycemia: Implications for the "monoaminehypothesis". Pharmacopsychiatria 1981;14:100-6.

5. Mueller PS, Heninger GR, McDonald RK. Intravenous glucose tolerance test in depression. Arch Gen Psychiatry 1969;21:470-7.

6. Heninger GR, Mueller PS, Davis LS. Depressive symptoms and the glucose tolerance test and insulin tolerance test. J Nerv Ment Dis 1975;161:421-32.

7. Talaei A, Siavash M, Majidi H, Chehrei A. Vitamin B12 may be more effective than nortriptyline in improving painful diabetic neuropathy. Int J Food Sci Nutr 2009;60(Suppl 5):71-6.

8. Sindrup SH, Tuxen C, Gram LF, Grodum E, Skjold T, Brøsen K, et al. Lack of effect of mianserin on the symptoms of diabetic neuropathy. Eur J Clin Pharmacol 1992;43:251-5.

9. Weber-Hamann B, Gilles M, Lederbogen F, Heuser I, Deuschle M. Improved insulin sensitivity in 80 nondiabetic patients with MDD after clinical remission in a double-blind, randomized trial of amitriptyline and paroxetine. J Clin Psychiatry 2006;67:1856-61 .

10. Khazaie H, Rahimi M, Tatari F, Rezaei M, Najafi F, Tahmasian M. Treatment of depression in type 2 diabetes with Fluoxetine or Citalopram? Neurosciences (Riyadh) 2011;16:42-5.
11. Kauffman RP, Castracane VD, White DL, Baldock SD, Owens R. Impact of the selective serotonin reuptake inhibitor citalopram on insulin sensitivity, leptin and basal cortisol secretion in depressed and non-depressed euglycemic women of reproductive age. Gynecol Endocrinol 2005;21:129-37.

12. Goodnick PJ, Kumar A, Henry JH, Buki VM, Goldberg RB. Sertraline in coexisting major depression and diabetes mellitus. Psychopharmacol Bull 1997;33:261-4.

13. Echeverry D, Duran P, Bonds C, Lee M, Davidson MB. Effect of pharmacological treatment of depression on $\mathrm{A} 1 \mathrm{C}$ and quality of life in low-income Hispanics and African Americans with diabetes: A randomized, double-blind, placebo-controlled trial. Diabetes Care 2009;32:2156-60.

14. Lustman PJ, Clouse RE, Nix BD, Freedland KE, Rubin EH, McGill $\mathrm{JB}$, et al. Sertraline for prevention of depression recurrence in diabetes mellitus: A randomized, double-blind, placebocontrolled trial. Arch Gen Psychiatry 2006;63:521-9.

15. Briscoe VJ, Ertl AC, Tate DB, Davis SN. Effects of the selective serotonin reuptake inhibitor fluoxetine on counterregulatory responses to hypoglycemia in individuals with type 1 diabetes. Diabetes 2008;57:3315-22.

16. Karabacak IY, Karabacak O, Toruner FB, Akdemir O, Arslan $M$. Treatment effect of sibutramine compared to fluoxetine on leptin levels in polycystic ovary disease. Gynecol Endocrinol 2004;19:196-201.

17. Ye Z, Chen L, Yang Z, Li Q, Huang Y, He M, et al. Metabolic effects of fluoxetine in adults with type 2 diabetes mellitus: $A$ meta-analysis of randomized placebo-controlled trials. PLoS One 2011;6:e21551.

18. Crucitti A, Zhang Q, Nilsson M, Brecht S, Yang CR, Wernicke J. Duloxetine treatment and glycemic controls in patients with diagnoses other than diabetic peripheral neuropathic pain: $A$ meta-analysis. Curr Med Res Opin 2010;26:2579-88.

19. Chen YC, Lin WW, Chen YJ, Mao WC, Hung YJ. Antidepressant effects on insulin sensitivity and proinflammatory cytokines in the depressed males. Mediators Inflamm 2010;2010:573594.

20. Hennings JM, Schaaf L, Fulda S. Glucose metabolism and antidepressant medication. Curr Pharm Des 2012;18:5900-19.

21. Laimer M, Kramer-Reinstadler K, Rauchenzauner M, LechnerSchoner T, Strauss R, Engl J, et al. Effect of mirtazapine treatment on body composition and metabolism. J Clin Psychiatry 2006;67:421-4.

22. Alderman CP, Condon JT, Gilbert AL. An open-label study of mirtazapine as treatment for combat-related PTSD. Ann Pharmacother 2009;43:1220-6.

23. Lustman PJ, Freedland KE, Griffith LS, Clouse RE. Fluoxetine for depression in diabetes: A randomized double-blind placebocontrolled trial. Diabetes Care 2000;23:618-23.

24. Lustman PJ, Williams MM, Sayuk GS, Nix BD, Clouse RE. Factors influencing glycemic control in type 2 diabetes during acute- and maintenance-phase treatment of major depressive disorder with bupropion. Diabetes Care 2007;30:459-66.

25. Katon WJ, Von Korff M, Lin EH, Simon G, Ludman E, Russo J, et al. The Pathways Study: A randomized trial of collaborative care in patients with diabetes and depression. Arch Gen Psychiatry 2004;61:1042-9.

26. Jones GR, Lazarus JH, Davies CJ, Greenwood RH. The effect of short term lithium carbonate in Type II diabetes mellitus. Horm Metab Res 1983;15:422-4.

27. Stephen LJ, Kwan P, Shapiro D, Dominiczak M, Brodie MJ. Hormone profiles in young adults with epilepsy treated with sodium valproate or lamotrigine monotherapy. Epilepsia 2001;42:1002-6.

28. York DA, Singer L, Thomas S, Bray GA. Effect of topiramate on body weight and body composition of osborne-mendel rats 
fed a high-fat diet: Alterations in hormones, neuropeptide, and uncoupling-protein mRNAs. Nutrition 2000;16:967-75.

29. Addis A, Koren G. Safety of fluoxetine during the first trimester of pregnancy: A meta-analytical review of epidemiological studies. Psychol Med 2000;30:89-94.

30. Cohen LS, Heller VL, Bailey JW, Grush L, Ablon JS, Bouffard SM. Birth outcomes following prenatal exposure to fluoxetine. Biol Psychiatry 2000;48:996-1000.

31. McElhatton PR, Garbis HM, Elefant E, Vial T, Bellemin B, Mastroiacovo $\mathrm{P}$, et al. The outcome of pregnancy in 689 women exposed to therapeutic doses of antidepressants. A collaborative study of the European Network of Teratology Information Services (ENTIS). Reprod Toxicol 1996;10:285-94.

32. Ericson A, Kallen B, Wiholm B. Delivery outcome after the use of antidepressants in early pregnancy. Eur $\mathrm{J}$ Clin Pharmacol 1999;55:503-8.

33. Kallen B, Otterblad Olausson P. Antidepressant drugs during pregnancy and infant congenital heart defect. Reprod Toxicol 2006;21:221-2.

34. Louik C, Lin AE, Werler MM, Hernandez-Diaz S, Mitchell AA. First-trimester use of selective serotonin-reuptake inhibitors and the risk of birth defects. N Engl J Med 2007;356:2675-83.

35. Alwan S, Reefhuis J, Rasmussen SA, Olney RS, Friedman JM; ; National Birth Defects Prevention Study. Use of selective serotonin-reuptake inhibitors in pregnancy and the risk of birth defects. N Engl J Med 2007;356:2684-92.

36. Hendrick V, Altshuler L. Management of major depression during pregnancy. Am J Psychiatry 2002;159:1667-73.

37. Nulman I, Rovet J, Stewart DE, Wolpin J, Gardner HA, Theis $\mathrm{JG}$, et al. Neurodevelopment of children exposed in utero to antidepressant drugs. N Engl J Med 1997;336:258-62.

38. Yonkers KA, Wisner KL, Stewart DE, Oberlander TF, Dell DL, Stotland $\mathrm{N}$, et al. The management of depression during pregnancy: A report from the American Psychiatric Association and the American College of Obstetricians and Gynecologists. Gen Hosp Psychiatry 2009;31:403-13.

39. Yonkers KA, Wisner KL, Stowe Z, Leibenluft E, Cohen L, Miller L, et al. Management of bipolar disorder during pregnancy and the postpartum period. Am J Psychiatry 2004;161:608-20.

40. Slone D, Siskind V, Heinonen OP, Monson RR, Kaufman DW, Shapiro S. Antenatal exposure to the phenothiazines in relation to congenital malformations, perinatal mortality rate, birth weight, and intelligence quotient score. Am J Obstet Gynecol 1977;128:486-8.

41. Gentile S. Antipsychotic therapy during early and late pregnancy. A systematic review. Schizophr Bull 2010;36:518-44.

42. Jacobson SJ, Jones K, Johnson K, Ceolin L, Kaur P, Sahn D, et al. Prospective multicentre study of pregnancy outcome after lithium exposure during first trimester. Lancet 1992;339:530-3.

43. Kennedy D, Koren G. Valproic acid use in psychiatry: Issues in treating women of reproductive age. J Psychiatry Neurosci 1998;23:223-8.

44. Lanza di Scalea T, Wisner KL. Antidepressant medication use during breastfeeding. Clin Obstet Gynecol 2009;52:483-97.

45. Eberhard-Gran M, Eskild A, Opjordsmoen S. Use of psychotropic medications in treating mood disorders during lactation: Practical recommendations. CNS Drugs 2006;20:187-98.

46. Stowe ZN, Hostetter AL, Owens MJ, Ritchie JC, Sternberg K, Cohen LS, et al. The pharmacokinetics of sertraline excretion into human breast milk: Determinants of infant serum concentrations. J Clin Psychiatry 2003;64:73-80.

47. Franssen EJ, Meijs V, Ettaher F, Valerio PG, Keessen M, Lameijer W. Citalopram serum and milk levels in mother and infant during lactation. Ther Drug Monit 2006;28:2-4.

48. Chaudron LH, Schoenecker CJ. Bupropion and breastfeeding: A case of a possible infant seizure. J Clin Psychiatry 2004;65:881-2.

49. Kohen D. Psychotropic medication and breast-feeding. Adv Psychiatr Treat 2005;11:371-9.

50. Barnas C, Bergant A, Hummer M, Saria A, Fleischhacker WW. Clozapine concentrations in maternal and fetal plasma, amniotic fluid, and breast milk. Am J Psychiatry 1994;151:945.

51. Chaudron LH, Jefferson JW. Mood stabilizers during breastfeeding: A review. J Clin Psychiatry 2000;61:79-90.

52. Miller LJ. Use of electroconvulsive therapy during pregnancy. Hosp Community Psychiatry 1994;45:444-50.

How to cite this article: Balhara YS, Verma R, Kalra B. Pharmacological management of psychiatric disorders in pregnancy complicated by diabetics. J Soc Health Diabetes 2014;2:70-6.

Source of Support: Nil. Conflict of Interest: None declared. 